\title{
Speculation on population
}

\author{
Matters of Life and Death: \\ Perspectives on Public Health, \\ Molecular Biology, Cancer, and the \\ Prospects for the Human Race \\ by John Cairns \\ Princeton University Press: 1997. Pp. 257.

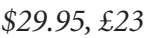

\section{JoelE.Cohen}

During his distinguished scientific career, John Cairns served as director of Cold Spring Harbor Laboratory on Long Island, New York, director of a laboratory of the Imperial Cancer Research Fund in London, and professor at Harvard School of Public Health in Cambridge, Massachusetts. He conducted and led research in molecular biology, cancer and public health. These three fields are the subject of two chapters each in this collection of six essays. The essays aim "to give a simple description of some of the great developments in the biological sciences, including the science that underlies the preservation of human health... the essays are designed to be read by people who know nothing about science".

Cairns achieves these goals. His lucid exposition shows how experiments, observations and calculations support some of the grand conclusions of modern biology. $\mathrm{He}$ offers fresh - although sometimes controversial — insights. I found the book an enjoyable way to learn more about my own and other fields.

Cairns's views on molecular biology and cancer are well known because of the wide attention given to his papers and his book Cancer: Science and Society (W. H. Freeman, 1978). At the editor's request, I therefore limit my comments to his views on mortality, population and the future of the human species, as presented in his first and last chapters.

Cairns's first chapter is a history of mortality. Worldwide, from the time of Palaeolithic hunter-gatherers to the middle of the eighteenth century, the average human life hardly exceeded 40 years anywhere. In seventeenth-century Breslau in Poland, as in Rome during the empire, most newborns did not survive long enough to have a child, and life expectancy was about 26 years.

In the eighteenth century, death rates began to fall, at first slowly and only among aristocracies. In subsequent centuries, the fall in death rate spread around the world and accelerated notably after the Second World War. Global life expectancy rose to 64 years by 1990-95, and as high as 80 years in Japan.

Why did the fall in death rates begin in the eighteenth century? Cairns mentions various theories: an increased consciousness of death rates stimulated by then-new statistical data and methods; an increased concern by governments for the welfare of the population as a basis for taxation and military strength; better nutrition; a declining virulence of infectious diseases; and a host of other improvements under human control such as vaccination, medical hygiene, increased trade, greater crop diversity, better buildings that excluded rats with plague, more washable fabrics for clothes, and declining fatalism about the lot of humans. From the very beginning and still today, within and among nations, the poor suffered higher death rates than the rich.

Cairns offers provocative speculations about the future of mortality. "For the rich nations it seems unlikely that there can be much more change in the pattern of mortality, because they have already attained a life expectancy that takes most of the population to the brink of senility. What these countries now have to do is learn how to run a nation where 25 per cent of the population are receiving pensions and expensive health care and are too old to work, 20 per cent are too young to work, and there is less and less work for most of the remaining 55 per cent." This forecast of little change reminds me of the remark in 1972 by Sir Macfarlane Burnet and David White that "the most likely forecast about the future of infectious disease is that it will be very dull".

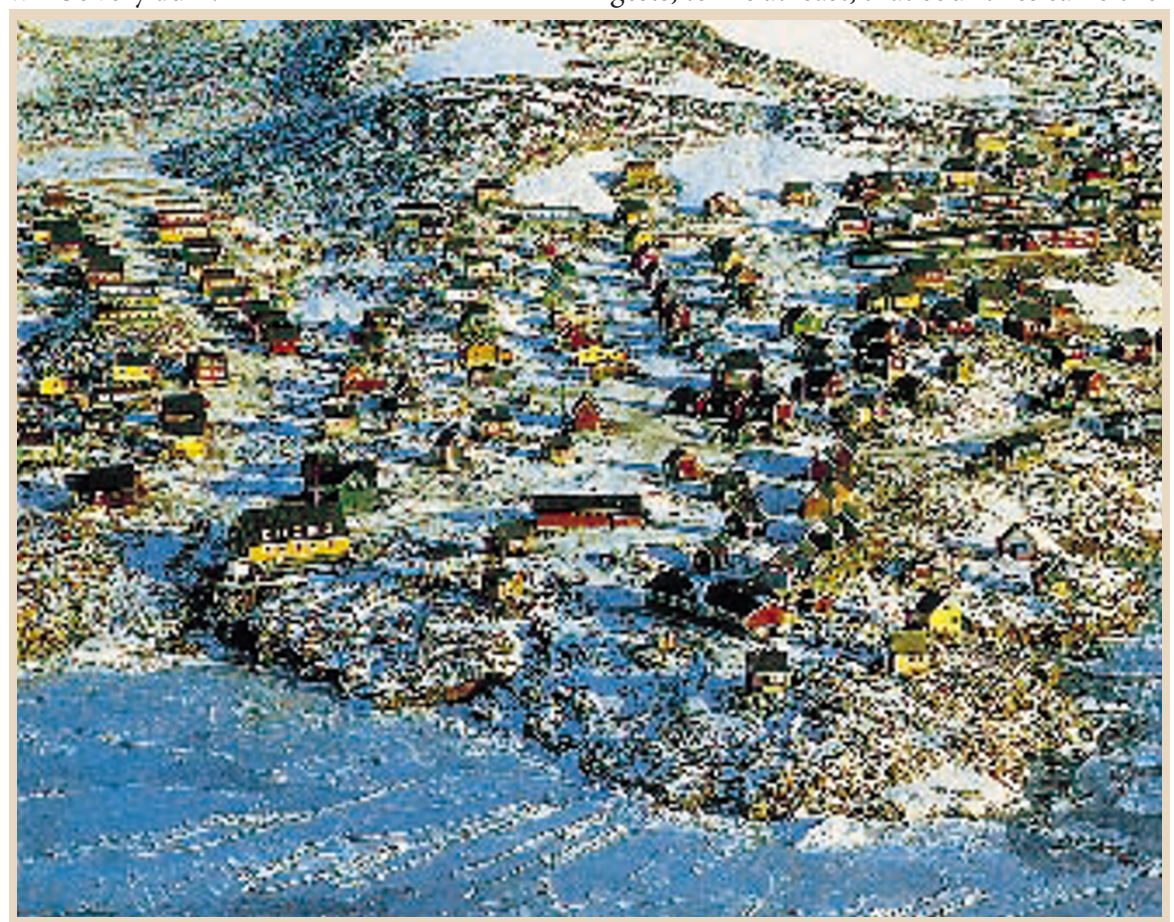

\section{Bleak houses}

"Beneath Scoresbysund's benign appearance from the air is a wild native village with high rates of violent crime, alcoholism, and suicide." This view of a tarnished wilderness in Greenland is one of many striking

Mortality change in today's poor countries is unlikely to retrace the path of mortality in the now-rich countries, Cairns argues, because poor countries are experiencing an unprecedented speed and magnitude of demographic change, because the tropical countries have a greater abundance of potential vectors of infectious diseases, and because the poor countries have to suffer advice and interventions from the rich.

Cairns illustrates this last problem with a startling graph taken from a 1983 paper by J. T. Hart. For 19 industrialized countries, the figure shows the number of doctors per 10,000 population on the abscissa and, on the ordinate, the residual of infant mortality after adjusting for a simple linear relationship with gross national product (GNP) per person. "You might guess, for example, that the more doctors you have, the lower will be infant mortality" after adjusting for GNP per person. The data demonstrate the opposite.

Japan and Finland in the lower left corner have the smallest number of doctors per person and the lowest adjusted infant mortality; Italy, West Germany and Austria in the upper right corner are highest on both variables.

"This does not, of course, mean that doctors are a cause of death in babies. Rather it suggests, to me at least, that countries can either r 
manage infant and maternal care at the national level (which is mainly a matter of prenatal care and does not require advanced technology) or they can choose to wash their hands of the whole thing and let market forces determine what happens. And market forces will lead to an overabundance of doctors... we should advise developing countries to go for the inexpensive forms of public health... rather than [imitate] Italy and West Germany."

This interpretation, while plausible, is not conclusive. Would infant mortality (adjusted for GNP per person) decline, as Cairns predicts, if public health expenditures or the fraction of infants who received prenatal care were plotted on the abscissa instead of doctors per person? If infant mortality were adjusted for other variables in addition to GNP per person, would the relationship with the number of doctors per person change? It seems difficult to argue with Cairns's general conclusion: "The most important message of this chapter is simply that whenever statistics are available, it is folly not to use them." But I would add the proviso, "carefully".

Cairns's final chapter, "Population", has three parts. The first reports how Malthus's views on the relative importance of the positive and preventive checks on population evolved as he collected relevant data. The second part is a brief history of "the explosion in the populations of Homo sapiens". In England, up to the beginning of the nineteenth century (just when Malthus was writing), population and prices moved hand in hand. As the number of people increased, the cost of living increased proportionately. So population growth led to scarcity of resources. As population and prices rose, life expectancy fluctuated erratically between 35 and 40 years. So the Malthusian view that rising population would call forth positive checks (rising death rates) in response to scarcity was not true for England up to the time Malthus wrote. Rather, rates of marriage changed, thereby adjusting fertility in response to changes in wages (a preventive check).

Between 1750 and 1850, England, Sweden and France experienced completely different trajectories of birth and death rates. These different responses to different social and economic circumstances, even within the cultural sphere of Western Europe, make the search for universal models of demographic change much more difficult.

The third part of the chapter deals with "Climate and the long-term prospects for the human race". Cairns gives examples to "suggest that all the technology in the world may be hard pressed to protect us from the effects of a really massive change in climate. What Malthus might have called the ultimate danger is that the Earth's climate may, for all we know, be delicately poised and easily pushed in one direction or the other.... The worry, therefore, is that the changes we are causing in the Earth's atmosphere may unlock the Earth from its present accommodating pattern of climate."

Cairns reproduces a graph of average global temperature (inferred from ice cores) over the past 80,000 years. It shows that the stable warmth experienced over the past 10,000 years is exceptional. Cairns suggests that humans may rock the climatic boat, but another possibility is that the climatic boat may rock for reasons humans simply do not understand.

This final chapter is a curious mix of certainty and doubt, of optimism and gloom. It opens with a flat assertion: "Sooner or later every nation will have gone through the demographic transition and have adjusted to its new, reduced death rate by lowering its birth rate." I wonder how he knows that.

From 1987 to 1995, male life expectancy in Russia fell from 65 to 58 years as male death rates rose. Maurice King has forecast demographic entrapment, in which poor countries so degrade their natural environments that they are unable to lower death rates. Bernard Gilland has warned of the possibility of a demographic interlude, in which death rates fall and then, because birth rates do not fall rapidly enough, death rates rise again.

I think Cairns's forecast is quite likely, but hardly inevitable, because (I hope) both the wealthy and the poor will eventually see that it is in their self-interest to create the conditions that will make the demographic transition happen everywhere. For the world today, the demographic transition is a choice, not an inevitability.

On the very same page, Cairns jumps from the optimistic certainty of his opening sentence to doubtful gloom: "Unfortunately, as a result of the Industrial Revolution, humankind has acquired a heightened ability to consume resources, and this great proliferation.... will bequeath to the Earth as a whole a legacy from which it may never recover... [Unlike] the collapse of the Mayan civilization, this time the disaster promises to be on a global scale." I wonder how he knows that.

In discussing population, Cairns's tone is highly charged: "The way we despoil the world... is the product of two factors... human behavior [and] the number of humans whose behavior has to be modified. Yes, the average inhabitant of the Western world consumes too much; but that is bad for the Earth because there are already too many of these consumers and soon there are going to be many more." On reading these lines, some people will nod agreement, and some will shout denial, but the analytical question of what constitutes "too much" and "too many" has not been answered.

In another unanalysed claim, Cairns states a theory of human fertility: "Like many animals, humans tend to adjust their rate of reproduction to match what they believe will be the opportunities for the next generation."
There is no supporting citation. I wonder how he knows that.

Scientists value conceptual analysis and factual support for their own sake. A broader social value of analysis and facts is that they provide credible common ground for moving towards consensus and action. The Cato Institute (on the 'population right') and Negative Population Growth (on the 'population left') can agree that the 1.2 billion people in the world with 1995 average GNP per person of US\$19,300 have a population doubling time, at 1997 growth rates, of around 560 years, while the other 80 per cent of the world's population (4.5 billion), with 1995 average GNP per person around US $\$ 1,100$, would double in 38 years at 1997 growth rates. They can agree that more people eat better now than ever before, but that as many as 2 billion are malnourished and 0.8 billion are undernourished.

They can agree that infant mortality rates globally are lower than ever before, but a newborn in the poor world has a chance of dying before its first birthday about seven times higher than that of a newborn in the rich world. They can agree that more people are literate than ever before, but that approximately a billion are illiterate and two-thirds of those are women.

Neither complacency nor alarm can replace factual analysis of the interactions among populations, economics, the environment and cultures.

Joel E. Cohen is at the Laboratory of Populations, Rockefeller University and Columbia University, 1230 York Avenue, Box 20, New York, NY 100216399, USA

(email:cohen@rockvax.rockefeller.edu).

\section{Nostalgia for the USSR}

\section{Essays of a Soviet Scientist: A Revealing Portrait of a Life in Science and Politics}

by Vitalii I. Gol'danskii

AIP Press: 1997. Pp. 303. $\$ 32.95, \mathfrak{£} 27.50$

Zhores A.Medvedev

Until recently, the former Soviet Union was perceived as an extremely centralized superpower. But within it there were parallel hierarchical pyramids of rank and profession in which people aspired to rise throughout their lives. The Academy of Sciences of the USSR was the highest and most stable of those pyramids, offering great privilege and honour to those at its apex.

Not only the most famous scientists but also those who were most loyal to the Soviet regime were elected to the ranks of the academy's 300 life members. In the past 50 years only one political dissident - Andrei Sakharov - emerged among the members 